
internationales

vol. 29 - $n^{\circ} 1 \mid 2013$

Mémoires et migrations en Afrique de l'Ouest et en France

\title{
Éditorial : Mémoires et migrations en Afrique de l'Ouest et en France
}

Editorial: Memories and Migrations in West Africa and France

Editorial: Memorias y migraciones en África Occidental y Francia

Marie Rodet et Christoph Reinprecht

\section{OpenEdition}

Journals

\section{Édition électronique}

URL : https://journals.openedition.org/remi/6250

DOI : $10.4000 /$ remi.6250

ISSN : $1777-5418$

Éditeur

Université de Poitiers

Édition imprimée

Date de publication : 1 mars 2013

Pagination : $7-22$

ISBN : 979-10-90426-07-8

ISSN : 0765-0752

Référence électronique

Marie Rodet et Christoph Reinprecht, «Éditorial : Mémoires et migrations en Afrique de l'Ouest et en France », Revue européenne des migrations internationales [En ligne], vol. 29 - $n^{\circ} 1$ | 2013, mis en ligne le 01 mars 2016, consulté le 15 avril 2022. URL : http://journals.openedition.org/remi/6250 ; DOI : https://doi.org/10.4000/remi.6250 


\section{Éditorial}

\section{Mémoires et migrations en Afrique de l'Ouest et en France}

\section{$\ldots$ Marie Rodet ${ }^{1}$ et Christoph Reinprecht ${ }^{2}$}

Jusque dans les années 1990, la thématique " mémoires et migrations " a surtout été abordée dans le cadre des Diaspora Studies anglo-saxonnes. La discussion s'est longtemps centrée sur la question de la diaspora africaine et de son identité en Amérique du Nord $^{3}$ qui a vu s'opposer les défenseurs d'un projet ontologique de la diaspora africaine (Echeruo, 1999) et les partisans du "Black Atlantic " ${ }^{4}$. Parallèlement, se sont également développées les Memory Studies. Intimement liées, au départ, à la question de la Shoah, celles-ci se sont progressivement étendues à tous les domaines des sciences sociales, avec une attention particulière portée à la question des traumatismes ${ }^{5}$.

L'analyse croisée entre migration, mémoire et traumatisme est ainsi demeurée, jusque dans les années 2000, I'apanage des recherches nord-américaines (Foner et Alba, 2010). Cette problématique s'est ensuite réinvitée en Europe avec notamment les débats autour de l'héritage de l'esclavage et de la colonisation; en France, elle a été abordée sous l'angle de la "question noire " (Thomas, 2006 ; Cottias, 2007 ; Ndiaye, 2009 ; Lozès et Lecherbonnier, 2009) et des " migrations postcoloniales " (Hajjat, 2006 ; Boubeker et Hajjat, 2008 ; Morokvasic-Muller, Dinh, Potot et Salzbrunn, 2008).

Alors que les mémoires de l'esclavage et de la colonisation avaient tendance en France à être oblitérées par l'injonction à « I'intégration » (Sayad, 1994 ; Cottias,

\footnotetext{
1 Lecturer in the History of Africa, History Department, School of Oriental and African Studies, University of London; mr28@soas.ac.uk

2 Professeur de sociologie, Institut für Soziologie, Universität Wien, Autriche ; christoph. reinprecht@univie.ac.at

3 L'histoire de la diaspora africaine en Europe est également un sujet de plus en plus étudié. De nombreuses études ont contribué à l'émergence d'une "Black History " européenne ces dix dernières années : voir notamment Dewitte (1985) : Blanchard, Deroo et Manceron (2001) ; Northrup (2002) ; Brent (2003); Earle et Lowe (2005) ; Thomas (2006) ; Noël (2006) ; Tardieu (2006) ; Gilroy (2007) ; Dabydeen, Gilmore et Jones (2007) ; Germain (2008) ; Gnammankou et Modzinou (2008) ; Blanchard (2011) ; Dedieu (2012).

4 Gilroy (2001) définit le " Black Atlantic " comme un espace culturel transnational construit à partir d'une longue histoire d'interconnections, d'échanges et d'influences mutuelles (" not roots, but routes ").
}

5 Nous tenons à remercier Martine Rodet, Illia Djadi, Caroline Roussy, Romain Tiquet et Cheikhna Wagué pour leur relecture attentive et leurs commentaires judicieux sur ce texte. 
2007 : 77-78 ; Favell, 1998 ; Hajjat, 2006 : 31-35, Laborde, 2001 ; Lucassen, 2005), le " retour des mémoires " s'est invité sur la scène politique, avec tout d'abord la loi dite "Taubira " de 2001 concernant la reconnaissance comme crime contre I'humanité des traites et des esclavages pratiqués à partir du XVe siècle sur certaines populations ; puis la promulgation de la loi du 23 février 2005 portant reconnaissance de la Nation et contribution nationale en faveur des Français rapatriés, et plus particulièrement son article 4 sur les aspects positifs de la colonisation. Le débat s'est particulièrement envenimé suite aux conflits sociaux qui éclatèrent dans les " banlieues françaises " à I'automne 2005 (Constant, 2009 ; Tshimanga, Gondola et Bloom, 2009), suivi par le discours dit " de Dakar " par Nicolas Sarkozy le 26 juillet 2007 et le débat sur l'identité nationale en France en 2009. Dans ce contexte, de nombreux mouvements politisés se sont mobilisés pour rappeler la nécessité d'un " devoir de mémoire " du passé colonial et esclavagiste français dans la lutte contre le racisme ${ }^{6}$. Cela n'a pas été non plus sans d'importants débats chez les historiens ${ }^{7}$.

Ces évènements ont permis une certaine "libération des mémoires " (Constant, 2007) qui s'est parfois transformée, comme le souligne Chivallon (2010), en une "frénésie mémorielle ", un " affolement commémoratif ", allant jusqu'à provoquer de véritables "guerres de mémoires " (Stora, 2007) au sujet de la construction de l'identité française et républicaine. Mais s'est également opérée une prise de conscience progressive, tant dans l'espace public que dans le champ universitaire, de processus "d'invisibilisation " (Dobie, 2004) des " mémoires subalternes " de la migration dans ses rapports avec I'histoire de l'esclavage et de la colonisation.

Comme le souligne Berliner (2005), on assiste désormais des deux côtés de l'Atlantique à un "boom mémoriel ". Celui-ci est sans doute également lié à la possibilité croissante offerte à un plus large public de se réapproprier le fait historique, possibilité encouragée en particulier par l'émergence de l'histoire "from below " et le développement de I'histoire sociale, I'histoire orale, la micro-histoire, I'histoire du genre et des femmes ou encore les Subaltern Studies. Ces courants historiographiques se sont tous intéressés d'une manière ou d'une autre à I'histoire des " invisibles " ; ceux que l'histoire politique et

6 On notera notamment le rôle joué par le Collectif des Antillo-Guyanais et Mahorais, le Conseil Représentatif des Associations Noires (CRAN), I'Appel des indigènes de la République ou encore le Collectif Devoir de Mémoire et la Brigade anti-négrophobie. Mais comme le rappelle Myriam Cottias (2007), plusieurs " questions noires " ont été liées sans distinction dans ce processus de réaffirmation politique du (post) colonial : I'histoire globale de la diaspora africaine dans les territoires et départements d'Outre-mer français est, sans aucun doute, spécifique à chacune de ces entités géographiques, de même qu'elle est différente de celle liée aux différents flux migratoires issus de chacune des anciennes colonies françaises en Afrique. La question de la mémoire coloniale en Afrique subsaharienne reste encore marginale dans ces débats qui ont essentiellement pour objet la mémoire de l'esclavage transatlantique et la mémoire de la colonisation en Algérie (sur ce dernier point, voir en particulier: Derderian, 2002 ; House et MacMaster, 2006 ; Hargreaves 2005 et 2007).

7 On citera la plainte, finalement retirée, déposée par le Collectif des Antillais, Guyanais, Réunionais contre I'historien Pétré-Grenouilleau qui avait montré dans ses travaux que la traite n'avait pas un caractère génocidaire, ou encore les nombreuses discussions sur les "guerres de mémoire " liées à la colonisation en Algérie, enfin les réponses sans équivoques des historiens au Discours de Dakar. Voir Pétré-Grenouilleau (2004) ; CoqueryVidrovitch (2009) ; Savarèse (2007) ; Stora (2007) ; Gassama (2008) ; Chrétien (2008) ; Ba Konaré (2008). 
globale dominante avait ignorés ou relégués au rang de simples victimes. On assiste donc à une " démocratisation du savoir historique " qui a permis en particulier de dépasser l'opposition entre mémoire et histoire ${ }^{8}$. Tout un chacun, désormais, est invité à participer à la revalorisation de l'histoire et des mémoires locales, le niveau national n'étant plus le seul lieu d'articulation des identités et des mémoires collectives (Misztal $2010: 26)^{9}$. II convient cependant de préciser que cette tendance à l'inflation mémorielle n'est pas sans poser problèmes. Elle entretient l'illusion qu'il en est désormais fini des processus de sélection, d'oubli et " d'invisibilisation ", mais c'est là méconnaître ce que soulignait Ricoeur (2004) : les processus de reconnaissance de certains faits historiques ne peuvent en général s'opérer sans des phénomènes parallèles d'oubli, voire pour d'autres auteurs de " tabou-isation " (" naming and shaming "). De son côté, Noiriel parlait paradoxalement de "boom mémoriel dans des sociétés amnésiques" (1995) évacuant la question du traumatisme et de la mémoire coloniale des débats sur l'histoire des migrations en Europe (Walkowitz et Knauer, 2004).

Depuis les années 2000, l'esclavage et la colonisation sont devenus les référents principaux dans les controverses mémorielles (Fouéré, 2010 : 8 ; Constant, 2007), non seulement en Europe et en Amérique, mais également de plus en plus en Afrique avec les demandes de réparation et la remise en cause des récits historiques énoncés exclusivement du point de vue des anciennes métropoles (Chrétien et Triaud, 1999).

En s'inscrivant dans ce contexte, l'ambition du dossier "Mémoires et migrations en Afrique de l'Ouest et en France " est de mettre en parallèle les mémoires dites "subalternes " de la migration. Par " mémoires subalternes ", nous entendons des mémoires construites, transformées, " tronquées " (Pandey, 2000 ; Rossi, 2009a), consciemment ou non, voire " traumatiques "(Araujo, 2009) qui sont matérialisées dans des milieux sociaux à caractère hégémonique qui tentent eux-mêmes de nier toute existence à ces mémoires subalternes.

Les différentes contributions rappellent comment les mémoires individuelles et collectives ne peuvent se former en dehors des structures hégémoniques passées et présentes (Prakash, 2000), qui, dans le contexte migratoire, tentent particulièrement de monopoliser les processus complexes d'oubli et de mémorialisation. Les tentatives hégémoniques de contrôle sont ici analysées dans deux espaces différents, mais liés en partie par l'expérience migratoire, coloniale et esclavagiste :

- d'une part, à partir de l'expérience des descendants d'esclaves en Afrique de l'Ouest qui, malgré les changements apportés par la décolonisation et la

\footnotetext{
8 Toutes les données historiques, qu'elles se manifestent dans les mémoires locales ou qu'elles soient accumulées dans des archives écrites, ont été produites dans un but précis, avoué ou non, conscient ou non ; elles ont toutes un dessein politique, que celui-ci soit arrêté dans le passé comme dans le cas des archives ou que celui-ci continue et soit réactivé, transformé, restructuré dans le présent comme dans le cas des mémoires locales (Rodet, 2010 : 267-268). La distinction souvent faite entre histoire et mémoire (Halbwachs, 1957 ; Nora, 1984 ; Le Goff, 1988) pour souligner la plus grande validité de I'une par rapport à I'autre est donc difficilement tenable (Burke 1989 ; Coquery-Vidrovitch 2006 ; Szpocinski 2009).

9 Selon Foner et Alba : " "Collective memory" refers to shared representations of the past, involving the meanings and forms - including speeches, monuments, scholarship, textbooks, and official ceremonies, through which the past is remembered and influences action in the present " (2010:800).
} 
démocratisation, sont encore stigmatisés par la politique mémorielle locale qui refuse d'intégrer leur histoire migratoire autrement que par le biais de la violence structurelle liée à I'histoire de l'esclavage (Paolo Gaibazzi et Lotte Pelckmans) ;

- d'autre part, à partir des expériences des migrants africains et de leurs descendants en France, dont les biographies et les mémoires semblent n'avoir que peu leur place dans la France postcoloniale, si ce n'est comme symboles d'un " échec mémoriel " - dès lors réutilisés par les groupes hégémoniques pour réaffirmer la légitimité de leur domination mémorielle (Lila Belkacem et Claire Lévy-Vroelant).

Alors que Spivak (1988) remet en cause la possibilité d'accès à ces mémoires subalternes, les articles de ce dossier s'intéressent plutôt à la signification pour les migrants et descendants de migrants des expériences mémorielles de l'esclavage et du fait colonial. Comment et dans quelles circonstances ces mémoires se forment, se transforment, se revivent et disparaissent ? Dans quelles circonstances les subalternes ont-ils finalement l'opportunité de se faire entendre et souhaitent-ils eux-mêmes s'exprimer dans un contexte politique donné ? Les articles prennent ainsi en compte les rapports de force en place au moment de la mobilité et au moment où ces mémoires ont pu être produites, transmises, restituées, ou bien tues.

La mise en parallèle des situations migratoires en Afrique de l'Ouest et en France nous invite à repenser les rapports complexes entre mémoire et migration en termes de " milieux de mémoire " plutôt que de "lieux de mémoire "(Nora, 1984), quand bien même les uns ne sont pas exclusifs des autres. II s'agit donc d'appréhender la signification des mémoires subalternes pour un milieu social particulier où le passé est porté et réactivé au quotidien par ses acteurs, de manière à la fois discursive et non-discursive. Dans cette perspective, les sciences sociales s'intéressent moins aux questions des normes et des valeurs intériorisées et partagées qu'à celles de la " performativité " des pratiques sociales (Olick et Robbins, 1998 ; Fortier, 1999). La formation de I'appartenance à un groupe à travers la mémoire (dans remembering on retrouve la notion de member) serait ainsi reconnaissable dans les corps. Comme le montre l'ensemble des contributions à ce dossier, les mobilités s'avèrent de véritables memoryscapes ${ }^{10}$ "performés " des rapports sociaux et politiques, tant dans les milieux d'origine que dans ceux d'arrivée.

Une telle analyse doit nous encourager à dépasser le cadre national des anciennes métropoles et à " provincialiser l'Europe " (Chakrabarty, 2000) en s'interrogeant, d'une part, sur la manière dont ces questions mémorielles font partie intégrante en Afrique des relations sociales des pays concernés (Fouéré, 2010) et d'autre part, sur la façon dont ces logiques continuent d'accompagner et d'être mobilisées par les migrants eux-mêmes dans leurs trajectoires migratoires régionales et transnationales.

10 Argenti et Röschenthaler définissent " memoryscapes " comme des " bodies of cultural memory transposed through space and place, and contested by different communities in a cultural palimpsest or shibboleth that belongs at once to many places, times and peoples, and which is forever to be re-inscribed, re-imagined and [...] re-lived " (2006: 40). 
II s'agit également de comprendre les mouvements migratoires et les questions mémorielles dans une perspective d'intersectionalité : les groupes migratoires sont loin d'être homogènes. Ils s'articulent en termes de constructions sociales de race, d'ethnicité, d'âge, de genre ${ }^{11}$, de génération, de classe sociale, etc. Se souvenir et réaffirmer une appartenance se fait en effet à travers des corps ethnicisés et genrés alors même que la mobilité de ces corps ethnicise et " genrifie " également l'espace revendiqué (Fortier, 1999 : 59). Les discours politiques nationaux sur les migrations tendent aussi à homogénéiser et essentialiser ces populations dans une lecture ethnique (on parle de " minorités ethniques ") et genrée de la différence (Winter, 1994 ; Phillips, 1995 ; Freedman et Tarr, 2000 ; Andall, 2003 ; Sargent et Cordell, 2003 ;Trica, 2006).

\section{Mémoires nationales, lieux de mémoire et eurocentrismes}

Les débats en Europe sur "mémoires et migrations " se sont principalement intéressés ces deux dernières décennies aux manières dont était représentée I'histoire des migrations, des migrants, et des " minorités ", et surtout des anciens colonisés et de leurs descendants, dans les expositions, les musées et les livres, dans les médias et de manière générale dans les récits sur l'identité nationale et les migrations (Peabody et Stovall, 2003 ; Hale, 2008 ; Hintermann et Johansson, 2010)12. Mais dans cette " exposition " de mémoires orchestrées par les "entrepreneurs de mémoire ", il ne s'agit pas en réalité d'une simple restitution du savoir historique, mais bel et bien de définir une mémoire collective spécifique qui organise les identités de groupe et leurs frontières et se revendique souvent elle-même gardienne de la différence (Dubois, 2000). Cette mise en scène de la mémoire collective influence également les relations entre les différents groupes sociaux et ethniques.

Dans les pays dits " d'immigration " comme les États-Unis, les récits migratoires sont à la fois à la source de l'idéologie nationale (dans le sens d'une " religion civique ") et participent de la construction des communautés. En témoignent des "lieux de mémoire " comme Ellis Island ou le musée Tenement à New York. Mais comment intégrer les mémoires migratoires dans le " memoryscape " national si ces mémoires, comme dans le cas de nombreux pays européens, sont associées à des processus de marginalisation sociale et économique, à des expériences d'exclusion sociale et d'isolation territoriale, à des vies en marge de la société, dans des lieux "fantômes " ? Les lieux attestant de l'histoire des migrations sont souvent " sans valeur patrimoniale " (Barou, $2000: 3$ ). Ils témoignent surtout de la souffrance d'une " double absence " (Sayad, 1999) que les acteurs eux-mêmes et leurs descendants souhaitent souvent oublier, de

11 Comme le font remarquer Pessar et Mahler (2003) pour les migrations transnationales et Rodet (2009) pour les migrations africaines, les migrations ont encore du mal à être pensées dans une perspective genrée.

12 La Cité nationale de I'histoire de l'immigration (CNHI) à Paris vient ainsi d'organiser en novembre 2012 une conférence sur le thème Memories of migrations and historical time. 
même que les pouvoirs publics ${ }^{13}$. La restitution des mémoires dans ce cadre $\mathrm{n}^{\prime}$ est donc pas seulement une question politique et sociale, mais également une question éthique (Margalit, 2003 ; Reinprecht, 2013).

Les historiens des migrations en Europe ont depuis longtemps fait remarquer que, dans la plupart des pays européens, I'histoire de l'immigration, comme partie intégrante de l'histoire, nationale, avait en grande partie été ignorée. Or, cette amnésie aurait tendance à n'être comprise en France qu'à l'aune de la " crise de l'État-nation ", lequel apparaîtrait d'autant plus en crise que les mémoires nationales semblent de plus se transformer en des entités complexes cosmopolites sous l'effet de la mondialisation. Les États-nations se révèleraient ainsi affaiblis dans leur rôle de formation des " communautés imaginées " (Misztal, 2010 ; Anderson, 1983).

S'est donc mise en place toute une muséologie afin d'inclure l'État-nation dans le "global " et de réintégrer l'histoire migratoire dans l'histoire nationale, suivant notamment le modèle du musée américain d'Ellis Island (Green, 2007) ${ }^{14}$. Mais ce "devoir de mémoire ", cette " mémorialisation ", tend également à réifier le passé sous la forme d'écrits, de récits et de "lieux " spécifiques (Fouéré, $2010: 7$; Rautenberg, 2007 ; voir également Belkacem, dans ce dossier). Cette histoire " officielle " des migrations ne laisse finalement, dans l'espace public, que peu de place aux mémoires subalternes.

Afin d'éviter ces tensions récurrentes entre mémoires officielles et mémoires subalternes, Gupta invite à l'oubli (2005), seul garant d'une égalité citoyenne. Mais même dans le cas où cet oubli serait à envisager sous l'angle de la réconciliation, si les mémoires hégémoniques continuent à avoir le contrôle du fonctionnement des mémoires subalternes - car celles-ci se forment en leur sein - I'oubli proposé par Gupta risque avant tout de renforcer les inégalités. Face aux crises mémorielles, les demandes de reconnaissance et de " justice mémorielle " semblent donc seules permettre la réintégration progressive des mémoires subalternes dans le discours sur la nation (Misztal, 2010).

Malgré ces réajustements, le niveau national s'accommode encore difficilement des mémoires s'inscrivant dans des cadres régionaux ou transnationaux, familiaux ou communautaires. Ainsi, de nombreux sociologues et historiens continuent, malgré ces limites, à n'envisager les productions mémorielles qu'à travers le prisme national, or cette approche tend à exclure les mémoires subalternes.

Comme le rappelle Gaibazzi dans ce dossier, les entreprises mémorielles européennes et transatlantiques semblent difficilement transposables en Afrique. Celles-ci sont en effet trop attachées à un projet nationaliste spécifique. Aussi, elles ne continuent à penser les mémoires diasporiques que comme contre-hégémoniques, alternatives, alors même que celles-ci se forment au sein du discours hégémonique.

13 Comme le fait remarquer Sibley (1995), les pouvoirs publics sont souvent responsables de la monopolisation de certains espaces et de la relégation de groupes dits "plus faibles " dans des environnements moins désirables. Voir également Bunnell and Nah (2004).

14 Voir en particulier la création de la CNHI à Paris en 2006. 
Mais au-delà de la transférabilité ou non de tels modèles, les expériences régionales et transnationales venant du continent africain semblent surtout nous inviter à repenser nos cadres eurocentriques et nationaux d'analyse des productions mémorielles et à appréhender ces mémoires avant tout à partir de leur signification pour des milieux de mémoire passés et présents, à l'heure où la mondialisation fait désormais partie intégrante de l'expérience locale des populations (Levy et Sznaider, 2002 : 87). Ainsi se créent-ils des milieux de mémoire mobiles et mobilisés (Belkacem et Pelckmans, dans ce dossier), entre local et global, alors même que les cadres classiques de la mémoire, que l'on continue à vouloir encore systématiquement ancrer dans un lieu géographique, se révèlent évanescents (Lévy-Vroelant, dans ce dossier). II faut dès lors envisager la question des mémoires migratoires dans un système de démocratie et de justice de plus en plus post-national (Misztal, 2005).

Pour retrouver ces mémoires subalternes, il importe désormais de comprendre comment celles-ci sont articulées et (im)mobilisées dans ces milieux de mémoire particulier. Cette réflexion sur le mode de production des mémoires n'en est encore qu'à ses débuts en sciences sociales alors qu'elle a été particulièrement favorisée par d'autres formes de production du savoir, en particulier la littérature ${ }^{15}$ (Arndt et Spitczok von Brisinski, 2006 ; Lévy-Vroelant, dans ce dossier), le cinéma ${ }^{16}$ et les arts plastiques ${ }^{17}$.

\section{Raconter les migrations, mobiliser les mémoires}

Si les Literary Studies se sont intéressées les premières à la place et à la voix des migrants, le recours aux récits de vie comme réservoir des expériences et des mémoires des migrants s'est progressivement étendu à l'ensemble des sciences sociales ${ }^{18}$ (Ireland et Proulx, 2001 ; Fouéré, 2010). Elles s'intéressent notamment à la manière dont les migrants parlent de leur expérience, si ces récits diffèrent de la mémoire collective, s'ils peuvent être lus comme des récits contre-hégémoniques, s'ils remettent en cause les discours sur les migrations, mais également s'ils peuvent être, et comment, réintégrés dans les récits collectifs sur les migrations.

15 La littérature des migrants fait de plus en plus l'objet de conférences scientifiques : voir la conférence Les migrations subsahariennes : par-delà les clichés, des richesses à partager, qui consacrait en janvier 2011 une journée aux vitalités artistiques des migrations, notamment à travers l'écriture, ou encore la conférence Ecritures en migration(s) tenue à Paris en mai 2012.

16 De nombreux films documentaires en particulier ont été produits en France ces dernières années pour replacer les voix des migrants et de leurs descendants au cœur de l'espace public: Musulmans de France de 1904 à nos jours (2009); Mémoires d'immigrés (2011) ; Correspondances (2011) ; Noirs de France (2012).

17 L'association AIDDA photographie et monte des expositions sur les quartiers d'immigration de Paris, comme la Goutte d'or, ainsi que d'autres sites et sujets liés à cette thématique depuis les années 1980. Elle publie ces photos dans une revue, Images \& Mémoires depuis les années 1990.

18 De nombreuses publications, destinées surtout au grand-public, notamment en France, se sont ainsi multipliées depuis les années 1990, allant de l'autobiographie au recueil de témoignages, voir parmi elles: Hawa/Vigor (1991) ; Cissé (1999) ; Les Paris des Africains (2002); Diakité et M’Dela-Mounier (2008) ; Bribes de mémoire (2012) ; Hathroubi-Safsaf (2012); Cordell et Sargent (2012). 
Ce qui reste cependant encore rarement abordé dans ces recherches est de savoir comment les questions sur les expériences et les mémoires migratoires sont posées aux migrants et à leurs descendants, par qui, et avec quelles conséquences. Ces questions sont fondamentales si on souhaite retrouver la position des migrants et de leurs descendants dans un milieu de mémoire particulier (Pelckmans et Belkacem, dans ce dossier).

Si ces mémoires subalternes ont une audience restreinte et restent peu diffusées dans l'espace public, elles témoignent pourtant de la production d'une communauté, de l'appropriation de ses normes et valeurs, ainsi que des transformations qu'elle vit (Shils, 1981 ; Misztal, 2010 ; Hoerder, 2000). Ceci passe en particulier par une recomposition permanente des réminiscences du projet migratoire (Gaibazzi).

Ce besoin de narration et de mise en récit à des fins sociales permet aux générations d'une même communauté de donner sens aux évènements passés (Olick et Levy, 1997 : 932). Quand les acteurs disparaissent, ces pratiques discursives et non discursives deviennent des " sites of second-order memory " pour les générations suivantes (Winter, 2010). Selon Fouéré, ces milieux de mémoires sont bel et bien " politisés ", car " c'est dans la dynamique même de production mémorielle que se forgent des subjectivités morales et politiques " (2010: 18-19). Mais leur mode de restitution, souvent traumatique et non-discursif, demeure encore mal connu.

En France, la mobilisation politique des communautés émigrées n'est pas un phénomène nouveau ${ }^{19}$. Mais celle-ci se décline de plus en plus de manière transnationale avec les pays d'origine, notamment en Afrique, dans la continuité des processus de démocratisation des années 1990. C'est sans doute l'un des traits majeurs des récits migratoires depuis les années 2000 (Pelckmans, dans ce dossier ${ }^{20}$. Les milieux de mémoires entre Afrique et Europe créent de nouveaux rapports de forces entre les sociétés d'accueil (dont la plupart des chercheurs sont originaires) et les migrants et descendants de migrants. Les mémoires individuelles et familiales, mais également les " metamemories " de la colonisation et de l'esclavage, sont revécues et réécrites dans ce contexte (Ireland et Proulx, 2010). On retrouve la mise en récit de ces tensions non seulement dans les revendications au droit de mémoire dans l'espace public ${ }^{21}$, mais également dans les récits de vie plus intimes, les lettres, les photographies, etc.

Ces transmissions mémorielles " anonymes " témoignent de formes plus structurelles de violence et d'inégalités. Mais elles montrent également des formes particulières du "souvenir " et en particulier la possibilité de formation de milieux de mémoire. Comme le montre l'ensemble des articles de ce dossier, les mémoires subalternes des migrants et de leurs descendants témoignent

19 Voir la production scientifique de plus en plus importante depuis les années 1970 sur le sujet : Samuel (1978) ; Barou (1978) ; Dewitte (1985) ; Timera (1996) ; Quiminal (1997) ; Daum (1998) ; Freedman (2008) ; Pojmann (2008) ; Dedieu (2012).

20 Voir en particulier l'action de l'AME (Association Malienne des Expulsés), une association créée en 1996 à Bamako et qui organise l'accueil des arrivants et soutient leurs revendications dans le champ politique (Chappart et Lecadet, 2011).

21 Reading discute le "droit de mémoire " et dans quelle mesure il peut être considéré comme un droit humain (Reading, 2011). 
finalement de la capacité de résilience et de mobilisation de ces communautés face aux mémoires nationales hégémoniques (Fouéré, 2010).

Dans son article, Paolo Gaibazzi explore comment les descendants d'esclaves dans les communautés soninké de Gambie, un pays marqué par une longue histoire migratoire inscrite dans les traditions locales des communautés, n'ont pas la même position dans la mémoire diasporique locale. Ils continuent d'être stigmatisés pour leurs origines " incertaines ". Ils sont ainsi toujours considérés comme des "étrangers ". La mémoire de l'esclavage apparaît ici avant tout comme un "public secret " (Rossi, 2009b) systématiquement réactivé par le discours hégémonique qui réaffirme la place des groupes dominants dans le maintien et la régulation des relations sociales. Dans ces situations, les descendants d'esclaves ne remettent pas pour autant en cause les modes de production de la mémoire hégémonique - ils semblent en effet les avoir acceptés et intégrés - mais s'attaquent plutôt à leurs effets immédiats sur leur place dans la société concernée en essayant notamment d'effacer le stigmate de l'esclavage dans leur généalogie. Le peu de mobilité des milieux de mémoire de l'esclavage semble ici particulièrement renforcé par leur mobilisation continue par les descendants d'esclaves et de nobles dans le débat sur les origines.

Lotte Pelckmans explique pourquoi les mémoires de l'esclavage dans les communautés maliennes originaires de la région de Douentza sont moins à rechercher dans les discours explicites sur l'esclavage que dans les pratiques genrées de la migration elle-même. Pour les descendants d'esclaves fulbé, la mobilité spatiale n'est pas forcément synonyme de mobilité sociale. Les hiérarchies sociales et les milieux de mémoire liés à l'esclavage semblent en effet continuer d'accompagner les migrants dans leur nouveau milieu de vie à Bamako ou à Paris. Ils acquièrent même dans certains cas un nouveau poids idéologique dans la justification des relations de dépendance. Cela semble empêcher dès lors toute réelle mobilisation sociale autour de ces questions, sauf peut-être dans certains foyers parisiens. Pelckmans démontre en particulier comment les jeunes femmes de statut esclave sont contraintes, bien plus que les hommes, à se conformer au rôle qui leur est assigné, ou du moins à s'y conformer dans certaines occasions. La migration est dès lors une pratique genrée non-discursive de la mémoire, un " memoryscape " de l'histoire de l'esclavage.

L'article de Lila Belkacem s'intéresse à la question du rôle de la mémoire des origines dans le vécu des descendants de migrants ouest-africains en France. Comme le montre l'auteure dans sa recherche empirique au sein d'une équipe d'ethnopsychiatres en région parisienne, les origines sont invoquées, dans les consultations, à des fins de reconnaissance sociale, mais également politique. Les ethnopsychiatres vont ainsi utiliser la revalorisation des origines (indifférenciées) africaines pour tenter de faire réagir les jeunes concernés. Ces invocations mémorielles sont plus ou moins couronnées de succès, car leur réaction est souvent ambivalente, voire indifférente, face à ces injonctions à la mémoire des origines. La pratique thérapeutique tend à construire une identification positive du " sujet africain " pour lutter contre les structures hégémoniques postcoloniales qui continuent à assujettir socialement les descendants des migrants ouest-africains. Mais cette pratique thérapeutique ne semble pas pour autant réussir à se départir des modes de production hégémoniques des discours sur l'identité et la citoyenneté. Si les " milieux de mémoire " sont mobilisés, ils 
n'échappent pas pour autant à leur milieu de production.

Enfin, le texte de Claire Lévy-Vroelant s'attache à analyser dans quels cadres les " mémoires subalternes " de la migration sont produites et peuvent être entendues. Contrairement à l'image répandue des " hôtels meublés " comme des lieux de marginalisation "sans valeur patrimoniale ", Lévy-Vroelant analyse ces hôtels comme des milieux possibles de production et d'échange de mémoires subalternes. Les hôtels meublés sont ainsi décrits comme des milieux de mémoire, des lieux qui créent un espace d'hospitalité potentielle, de production et de rencontre de mémoires qui sont souvent ancrées dans des évènements douloureux comme la guerre d'Algérie. L'auteure tente ainsi de comprendre comment ces mémoires sont transmises alors même que les porteurs de ces mémoires tendent à disparaître et que ces milieux de mémoires apparaissent particulièrement instables à cause, notamment, de la mobilité sociale et géographique de ses acteurs et des processus de modernisation de I'habitat parisien. La littérature joue ici un rôle central dans la transmission et soulève la question de la possibilité de mobilisation de ces milieux de mémoire sans mise en récit.

L'objectif poursuivi avec la publication des quatre articles qui composent ce dossier était d'ouvrir quelques pistes de réflexion à partir de situations particulières qu'interrogent les auteurs en Afrique de l'Ouest et en France. Les descriptions et les analyses qu'ils nous livrent nous invitent, et peut-être encourageront des chercheurs, à revisiter tant au niveau local que transnational la problématique des "lieux et des milieux de mémoire ", qui s'avère d'une grande actualité aussi bien en Afrique qu'en Europe.

\section{Références bibliographiques}

Andall Jacqueline (Ed.) (2003) Gender and Ethnicity in Contemporary Europe, Oxford, Berg, $252 \mathrm{p}$.

Anderson Benedict (1983) Imagined Communities, Londres, Verso, 224 p.

Araujo Ana Lucia (2009) Introduction: The Slave Past in the Present, in Ana Lucia Araujo Ed., Living History: Encountering the Memory of the Heirs of Slavery, Newcastle upon Tyne, Cambridge Scholars Publishing, pp. 1-7.

Argenti Nicolas and Röschenthaler Ute (2006) Introduction: Between Cameroon and Cuba:Youth, slave trades and translocal memoryscapes, SocialAnthropology, 14 (1), pp. 33-47.

Arndt Susan and Spitczok von Brisinski Marek (Eds.) (2006) Africa, Europe and (Post)Colonialism: Racism, Migration and Diaspora in African Literatures, Bayreuth, Bayreuth University, 346 p.

Barou Jacques (2000) Lieux de mémoire de l'immigration, Écarts d'ldentité, Hors série, pp. 2-4.

Barou Jacques (1978) Travailleurs africains en France, rôle des cultures d'origine, Grenoble, Presses Universitaires de Grenoble, Publications orientalistes de France, $162 \mathrm{p}$. 
Berliner David (2005) The Abuses of Memory: Reflections on the Memory Boom in Anthropology, Anthropological Quarterly, 78 (1), pp. 197-211.

Blanchard Pascal (2011) La France noire, trois siècles de présences, Paris, Éditions La Découverte, 360 p.

Blanchard Pascal, Deroo Éric et Manceron Gilles (2001) Le Paris noir, Paris, Éditions Hazan, 242 p.

Boubeker Ahmed et Hajjat Adbelalli (2008) Histoire politique des immigrations (post)coloniales, Éditions Amsterdam, 317 p.

Brent Hayes Edwards (2003) The Practice of Diaspora. Literature, Translation, and the Rise of Black Internationalism, Cambridge, Harvard University Press, 416 p.

Bunnell Tim and Nah Alice (2004) Counter-global cases for place: contesting displacement in globalising Kuala Lumpur metropolitan area, Urban Studies, 41 (12), pp. 2447-2467.

Burke Peter (1989) History as Social Memory, in Thomas Butler Ed., Memory: History, Culture and the Mind, Oxford, Blackwell, pp. 97-113.

Chakrabarty Dipesh (2000) Provincializing Europe: Postcolonial Thought and Historical Difference, Princeton, Princeton University Press, 336 p.

Chappart Pascaline et Lecadet Clara (2011) Enfants d'immigrés, enfants d'expulsés, dans I'arène des luttes et des politiques, Journal des Africanistes, 81 (1), pp. 163-184.

Chivallon Christine (2010) L'explosion mémorielle aux Antilles françaises et la révélation de mémoires anonymes, Cahiers d'Études Africaines, L (1), pp. 235-261.

Chrétien Jean-Pierre (Éd.) (2008) L'Afrique de Sarkozy : Un déni d'histoire, Paris, Karthala, 203 p.

Chrétien Jean-Pierre et Triaud Jean-Louis (Éds.) (1999) Histoire d'Afrique : Les enjeux de mémoire, Paris, Karthala, 504 p.

Cissé Madjigène (1999) Parole de sans-papiers, Paris, La Dispute, 254 p.

Constant Fred (2009) Talking Race in Colour-blind France: Equality Denied, "Blackness" Reclaimed, in Darlene Clark Hine, Trica Danielle Keaton and Stephen Smal Eds., Black Europe and the African Diaspora, University of Illinois Press, pp. $145-160$.

Constant Fred (2007) Pour une lecture sociale des revendications mémorielles " victimaires ", Esprit, 2, pp. 105-116.

Coquery-Vidrovitch Catherine (2009) Enjeux politiques de I'histoire coloniale, Agone, $190 \mathrm{p}$.

Coquery-Vidrovitch Catherine (2006) Préface, in Doulaye Konaté, Travail de mémoire et construction nationale au Mali, Paris, L'Harmattan, pp. 5-34.

Cordell Dennis D. and Sargent Carolyn F. (2012) Samba Sylla (b. 1948), Doulo Fofanna (b. 1948 or 1949), and Djénébou Traore (b. 1972). The Colonies Come to France, in Dennis D. Cordell Ed., The Human Tradition in Modern Africa, Rowman \& Littlefield Publishers, pp. 249-266. 
Daum Christophe (1998) Les associations de Maliens en France : Migration, développement et citoyenneté, Paris, Karthala, 250 p.

Dabydeen David, Gilmore John and Cecily Jones (2007) Oxford Companion to Black British History, Oxford University Press, 562 p.

Diakité Tidiane et M'Dela-Mounięr Nathalie (2008) L'immigration n'est pas une Histoire sans paroles, Ploërmel, Éditions Les oiseaux de papier, 256 p.

Dubois Laurent (2000) La République Métissée: Citizenship, Colonialism, and the Borders of French History, Cultural Studies, 14 (1), pp. 15-34.

Dedieu Jean-Philippe (2012) La parole immigrée. Les migrants africains dans I'espace public en France (1960 - 1995), Paris, Klincksieck, 336 p.

Derderian Richard L. (2002) Algeria as a lieu de mémoire: Ethnic Minority, Memory and National Identity in Contemporary France, Radical History Review, 83, pp. 28-43.

Dewitte Philippe (1985) Les mouvements nègres en France, 1919-1939, Paris, L'Harmattan, $415 \mathrm{p}$.

Dobie Madeleine (2004) Invisible Exodus: The Cultural Effacement of Antillean Migration, Diaspora: A Journal of Transnational Studies, 12 (2/3), pp. 149-183.

Earle Thomas F. and Kate J. P. Lowe (Eds.) (2005) Black Africans in Renaissance Europe, Cambridge University Press, 417 p.

Echeruo Michael J. C. (1999) An African Diaspora: The Ontological Project, in Isidore Okpewho, Carole Boyce Davies and Ali A. Mazrui Eds., The African Diaspora. African Origins and New World Identities, Bloomington, Indiana University Press, pp. 3-18.

Favell Adrian (1998) Philosophies of Integration: Immigration and the Idea of Citizenship in France and Britain, Basingstoke, Palgrave, 320 p.

Foner Nancy and Richard Alba (2010) Immigration and the Legacies of the Past: The Impact of Slavery and the Holocaust on Contemporary Immigrants in the United States and Western Europe, Comparative Studies in Society and History, 52 (4), pp. 798-819.

Fortier Anne-Marie (1999) Re-Remembering Places and the Performance of Belonging(s), Theory Culture Society, 16 (2), pp. 41-64.

Fouéré Marie-Aude (2010) La mémoire au prisme du politique, Cahiers d'Études Africaines, L (1), 197, Jeux de mémoires, pp. 5-24.

Freedman Jane (2008) Women, Migration and Activism in Europe, Amnis. Revue de Civilisation Contemporaine Europes/Amériques, 8, [en ligne] consulté le 17/02/2013. URL : http://amnis.revues.org/604.

Freedman Jane et Tarr Carrie (2000) Women, Immigration and Identities in France, Oxford, Berg, $210 \mathrm{p}$.

Gassama Makhily (Éd.) (2008) L'Afrique répond à Sarkozy : Contre le discours de Dakar, Paris, éditions Phillippe Rey, 540 p.

Germain Felix (2008) For the Nation and for Work: Black Activism in Paris of the 1960s, in Wendy Pojmann Ed., Migration and Activism in Europe since 1945, Palgrave Macmillan, pp. 15-32. 
Gilroy Paul (2007) Black Britain - A Photographic History (introduction by Stuart Hall), London, Saqi, 320 p.

Gilroy Paul (1993) The black Atlantic: modernity and double consciousness, Cambridge, Harvard University Press, 261 p.

Gnammankou Dieudonné et Modzinou Yao (Éds.) (2008) Les Africains et leurs descendants en Europe avant le XXe siècle, Toulouse, MAT éditions, 419 p.

Green Nancy L. (2007) A French Ellis Island? Museums, Memory and History in France and the United States, History Workshop Journal, 63, pp. 239-253.

Gupta Dipankar (2005) Learning to Forget: The Anti-Memoirs of Modernity, Oxford, Oxford University Press, 272 p.

Hajjat Abdellali (2005) Immigration postcoloniale et mémoire, Paris, L'Harmattan, $150 \mathrm{p}$.

Halbwachs Maurice (1957) Les cadres sociaux de la mémoire, Paris, Presses universitaires de France, $211 \mathrm{p}$.

Hale Dana S. (2008) Races on Display. French Representations of Colonized Peoples, 1886-1940, Bloomington, Indiana University Press, 215 p.

Hargreaves Alec G. (2007) Multi-Ethnic France. Immigration, Politics, Culture and Society, London, Routledge, 265 p.

Hargreaves Alec G. (Ed.) (2005) Memory, Empire, and Postcolonialism: Legacies of French Colonialism, Lanham, Lexington, 258 p.

Hargreaves Alec G. (2001) Perceptions of Ethnic Difference in Post-War France, in Susan Ireland and Patrice J. Proulx Eds., Immigrant Narratives in Contemporary France, Westport, CT, Greenwood Press, pp. 7-22.

Hathroubi-Safsaf Nadia (2012) Immigrations plurielles, témoignages singuliers, Paris, Éditions Les points sur les i, 134 p.

House Jim and MacMaster Neil (2006) Paris 1961: Algerians, State Terror, and Memory, Oxford, Oxford University Press, 288 p.

Hintermann Christiane and Johansson Christina (Eds.) (2010) Migration and Memory, Representations of migration in Europe since 1960, Innsbruck, Studien Verlag, $224 \mathrm{p}$.

Hoerder Dirk (2000) Creating Societies. Immigrant Lives in Canada, Montreal, McGill-Queens University Press, 416 p.

Ireland Susan and Proulx Patrice J. (Eds.) (2001) Immigrant Narratives in Contemporary France, Westport, CT, Greenwood Press, 248 p.

Konaré Adame Ba (Ed.) (2008) Petit précis de remise à niveau sur I'histoire africaine à I'usage du président Sarkozy, Paris, La Découverte, 362 p.

Laborde Cécile (2001) The Culture(s) of the Republic: Nationalism and Multiculturalism in French RepublicanThought, PoliticalTheory, 29 (5), pp. 716735.

Levy Daniel and Sznaider Natan (2002) Memory Unbound: The Holocaust and the Formation of Cosmopolitan Memory, European Journal of Social Theory, 5 (1), pp. 87-106. 
Lozès Patrick et Bernard Lecherbonnier (2009) Les Noirs sont-ils des Français à part entière?, Paris, Larousse, 157 p.

Lucassen Leo (2005) The Immigrant Threat: The Integration of Old and New Migrants in Western Europe since 1850, Urbana, University of Illinois Press, $280 \mathrm{p}$.

Margalit Avishai (2003) The Ethics of Memory, Cambridge. Harvard University Press, $240 \mathrm{p}$.

Misztal Barbara A. (2010) Collective Memory in a Global Age: Learning How and What to Remember, Current Sociology, 58 (1), pp. 24-44.

Misztal Barbara A. (2005) Memory and Democracy, American Behavioral Scientists, 48 (10), pp. 1320-1339.

Morokvasic-Muller Mirjana, Dinh Bernard, Potot Swanie and Salzbrunn Monika (2008) Immigrant France: Colonial heritage, labour (im)migration and settlement, IDEA Working Papers, 2, $60 \mathrm{p}$.

Noël Erick (2006) Être noir en France au XVIIle siècle, Tallandier, 320 p.

Noiriel Gérard (1995) Immigration: Amnesia and Memory, French Historical Studies, 19 (2), pp. 367-380.

Nora Pierre (1984) Entre Mémoire et Histoire : la problématique des lieux, in Pierre Nora Éd., Les lieux de mémoire, tome 1 : La République, Paris, Gallimard, pp. 23-43.

Olick Jeffrey and Levy Daniel (1997) Collective Memory and Cultural Constraint: Holocaust Myth and Rationality in German Politics, American Sociological Review, 62 (6), pp. 921-926.

Olick Jeffrey and Robbins Joyce (1998) Social Memory Studies: From 'Collective Memory' to the Historical Sociology of Mnemonic Practices, Annual Review of Sociology, 24, pp. 105-140.

Pandey Gyanendra (2000) Voices from the Edge: The Struggle to Write Subaltern Histories, in Vinayak Chaturvedi Ed., Mapping Subaltern Studies and the Postcolonial, London, Verso, pp. 281-299.

Peabody Sue and Stovall Tyler (Eds.) (2003) The Color of Liberty. Histories of Race in France, Durahm, Duke University Press, 400 p.

Pesovar Zsofia (2012) Parcours de migrants, d'un état à l'autre, Bribes de mémoires, 4, Rezé, Centre Interculturel de Documentation.

Pessar Patricia R. and Mahler Sarah J. (2003) Transnational Migration: Bringing Gender, International Migration Review, 37 (3), pp. 812-846.

Pétré-Grenouilleau Olivier (2004) Les traites négrières. Essai d'histoire globale, Paris, Gallimard, 468 p.

Phillips Anne (1995) The Politics of Presence. The Political Representation of Gender, Ethnicity and Race, Oxford, Oxford University Press, 224 p.

Pojmann Wendy (Ed.) (2008) Migration and Activism in Europe since 1945, Palgrave Macmillan, $288 \mathrm{p}$.

Prakash Gyan (2000) The impossibility of subaltern history, Nepantla: Views from South, 1 (2), pp. 287-94. 
Quiminal Catherine (1997) Un réseau d'associations de femmes africaines, Hommes et Migrations, 1208, pp. 24-30.

Rautenberg Michel (2007) Les " communautés " imaginées de l'immigration dans la construction patrimoniale, Les Cahiers de Framespa, 3, [en ligne], consulté le 17/02/2013. URL : http://framespa.revues.org/274

Reading Anna (2011) Identity, memory and cosmopolitanism: The otherness of the past and a right to memory?, European Journal of Cultural Studies, 14 (4), pp. 370-394.

Reinprecht Christoph (2013) La vulnérabilité des travailleurs immigrés âgés : autonomie et vieillissement dans des conditions précaires, in Marc-Henri Soulet Éd., Vulnérabilité : de la fragilité sociale à l'éthique de la sollicitude, Fribourg, Academic Press Fribourg.

Ricœur Paul (2004) Memory, History, Forgetting, Chicago, The University of Chicago Press, $624 \mathrm{p}$.

Rodet Marie (2010) Mémoires de l'esclavage dans la région de Kayes, histoire d'une disparition, Cahiers d'Études Africaines, L (1), 197, Jeux de mémoires, pp. 263-291.

Rodet Marie (2009) Les migrantes ignorées du Haut-Sénégal, Paris, Karthala, 332 p.

Rossi Benedetta (2009a) Without History? Interrogating "Slave" Memories in Ader (Niger), Paper presented at the Conference Tales of Slavery: Narratives of Slavery, the Slave Trade and Enslavement in Africa, University of Toronto, 20-23 May 2009.

Rossi Benedetta (Ed.) (2009b) Reconfiguring Slavery: West African Trajectories, Liverpool, Liverpool University Press, 256 p.

Samuel Michel (1978) Le Prolétariat africain noir en France, Paris, Maspero, 262 p.

Sargent Carolyn and Cordell Dennis (2003) Polygamy, disrupted reproduction, and the state: Malian migrants in Paris, France, Social Science \& Medicine, 56 (9), pp. 1961-1972.

Sayad Abdelmalek (1999) La Double Absence. Des Illusions de l'émigré aux souffrances de l'immigré, Paris, Seuil, 437 p.

Sayad Abdelmalek (1994) Qu'est-ce que I'intégration ?, Hommes et Migrations, 1182, pp. 8-14.

Savarèse Éric (Éd.) (2008) L'Algérie dépassionnée : au-delà du tumulte des mémoires, Paris, Syllepse, 214 p.

Shils Edward (1981) Tradition, Chicago, IL, University of Chicago Press, 342 p.

Sibley David (1995) Geographies of Exclusion: Society and Difference in the West, London, Routledge, $224 \mathrm{p}$.

Spivak Gayatri Chakravorty (1988) Can the Subaltern Speak?, in Cary Nelson and Lawrence Grossberg Eds., Marxism and the Interpretation of Culture, Urbana, University of Illinois Press, pp. 271-313.

Szpocinski Andrzej (2009) Histoire et mémoire sociale, in Isidore Ndaywel È Nziem et Elisabeth Mudimbe-Boyi Éds., Images, mémoires et savoirs : Une histoire en partage avec Bogumil Koss Jewsiewicki, Paris, Karthala, pp. 223-230. 
Tardieu Marc (2006) Les Africains en France de 1914 à nos jours, Paris, Éditions du Rocher, $219 \mathrm{p}$.

Timéra Mahamet (1996) Les Soninké en France, d'une histoire à l'autre, Paris, Karthala, $244 \mathrm{p}$.

Thomas Dominic (2006) Black France: Colonialism, Immigration, and Transnationalism, Bloomington, Indiana University Press, 305 p.

Trica Danielle Keaton (2006) Muslim Girls and the Other France. Race, Identity Politics, \& Social Exclusion, Bloomington, Indiana University Press, 223 p.

Trica Danielle Keaton, Sharpley-Whiting T. Denean and Stovall Tyler (Eds.) (2012) Black France/France noire, The History and Politics of Blackness, Durham, Duke University Press, $344 \mathrm{p}$.

Tshimanga Charles, Gondola Didier and Bloom Peter J. (Eds.) (2009) Frenchness and the African Diaspora. Identity and Uprising in Contemporary France, Bloomington, Indiana University Press, $352 \mathrm{p}$.

Tou Konaré Kadia (2002) Les Paris des Africains, Paris, Cauris Éditions, 174 p.

Vigor Catherine et Soukouna Hawa (1991) Hawa : L'Afrique à Paris, Témoignage avec la collaboration de Catherine Vigor, Paris, Flammarion, 232 p.

Walkowitz Daniel and Knauer Lisa M. (Eds.) (2004) Memory and the Impact of Political Transformation in Public Space, Durham, Duke University Press, 336 p.

Winter Bronwyn (1994) Women, the Law, and Cultural Relativism in France: The Case of Excision, Signs, 19 (4), pp. 939-974.

Winter Jay (2010) Sites of Memory, in Susannah Radstone et Bill Schwarz Eds., Memory: Histories, Theories, Debates, New York, Fordham University Press, pp. 312-324. 